\section{Clinical Trial Protocol}

Check for updates

\section{OPEN ACCESS}

Received: Jul 9, 2019

Revised: Aug 18, 2019

Accepted: Aug 21, 2019

Correspondence to

Byoung-Gie Kim

Department of Obstetrics and Gynecology,

Samsung Medical Center, Sungkyunkwan

University School of Medicine, 81 Irwon-ro,

Gangnam-gu, Seoul 06351, Korea.

E-mail: bgkim@skku.edu

*Additional KGOG investigators who participated in this study are listed in the acknowledgments.

Copyright $($ 2019. Asian Society of Gynecologic Oncology, Korean Society of Gynecologic Oncology

This is an Open Access article distributed under the terms of the Creative Commons Attribution Non-Commercial License (https:// creativecommons.org/licenses/by-nc/4.0/) which permits unrestricted non-commercial use, distribution, and reproduction in any medium, provided the original work is properly cited.

ORCID IDs

Jung-Yun Lee (D)

https://orcid.org/0000-0001-7948-1350

Jae-Weon Kim (D)

https://orcid.org/0000-0003-1835-9436

Myong Cheol Lim (D)

https://orcid.org/0000-0001-8964-7158

sunghoon Kim (iD)

https://orcid.org/0000-0002-1645-7473

Hee Seung Kim (iD)

https://orcid.org/0000-0001-6876-8671

Chel Hun Choi (D)

https://orcid.org/0000-0002-0199-6669
A phase II study of neoadjuvant chemotherapy plus durvalumab and tremelimumab in advanced-stage ovarian cancer: a Korean Gynecologic
Oncology Group Study (KGOG 3046), TRU-D

\author{
Jung-Yun Lee $\left(\mathbb{1},{ }^{1}\right.$ Jae-Weon Kim $\mathbb{1},{ }^{2}$ Myong Cheol Lim $\left(\mathbb{1},{ }^{3}\right.$ Sunghoon Kim $\left(\mathbb{1},{ }^{1}\right.$ \\ Hee Seung Kim $\left(\mathbb{D},,^{2}\right.$ Chel Hun Choi $\mathbb{1}_{0},{ }^{4}$ Ju Yeon Yi $\left(\mathbb{0},{ }^{5}\right.$ Sang-Yoon Park $\left(\mathbb{D},{ }^{3}\right.$ \\ Byoung-Gie Kim $\mathbb{1 0}^{4}$, and on behalf of KGOG investigators*
}

'Department of Obstetrics and Gynecology, Institute of Women's Life Medical Science, Yonsei University College of Medicine, Seoul, Korea

${ }^{2}$ Department of Obstetrics and Gynecology, Seoul National University College of Medicine, Seoul, Korea ${ }^{3}$ Division of Tumor Immunology and Center for Clinical Trial, Center for Gynecologic Cancer, Research Institute and Hospital, National Cancer Center, Goyang, Korea

${ }^{4}$ Department of Obstetrics and Gynecology, Samsung Medical Center, Sungkyunkwan University School of Medicine, Seoul, Korea

${ }^{5} \mathrm{R}$ connect, Seoul, Korea

\section{ABSTRACT}

Background: A single-arm phase II study of neoadjuvant chemotherapy plus durvalumab and tremelimumab in the treatment of advanced-stage ovarian cancer has begun in Korea. We hypothesized that adding durvalumab (anti-programmed death-ligand 1 antibody) and tremelimumab (anti-cytotoxic T-lymphocyte-associated protein 4 antibody) to chemotherapy in treating this cancer can increase progression-free survival (PFS) with minimal effects on safety. Methods: During treatment, serial biopsies will be performed on pre-treatment, at interval debulking surgery and progression to identify immune biomarkers and changes in the tumor microenvironment. Patients with histologically confirmed stage IIIC/IV epithelial ovarian cancer are offered durvalumab, tremelimumab plus chemotherapy for neoadjuvant chemotherapy and durvalumab plus chemotherapy for adjuvant chemotherapy. Twenty-four patients will be included from four Korean institutions within 1 year. The primary endpoint is a 12-month PFS rate.

Trial Registration: ClinicalTrials.gov Identifier: NCT03899610

Keywords: Epithelial Ovarian Cancer; Chemotherapy; Durvalumab; Tremelimumab; Immunotherapy 
Ju Yeon Yi (iD

https://orcid.org/0000-0002-4423-1076 Sang-Yoon Park (iD)

https://orcid.org/0000-0001-8325-6009

Byoung-Gie Kim (iD

https://orcid.org/0000-0002-0572-8450

Trial Registration

ClinicalTrials.gov Identifier: NCT03899610

Funding

This work is supported by AstraZeneca and by a faculty research grant of Yonsei University College of Medicine for 6-20180169. Research grants are administered by the Ministry of Science, ICT \& Future Planning (2017M3A9E8029714).

Conflict of Interest

Jung-Yun Lee received honoraria for speaker's bureaus from AstraZeneca, Janssen, Roche and research fund from AstraZeneca, Clovis Oncology, Janssen, MSD, Roche for clinical trials or contracted research. Jae-Weon Kim has served on local advisory boards for AstraZeneca, Takeda, Pfizer, Janssen. Received honoraria for speaker's bureaus from AstraZeneca, Takeda, Pfizer, Janssen, Roche. Myoung Chel Lim disclose COI with following companies on the one of the advisory board member, honoraria, research funding or travel for the meeting (AstraZeneca, Cellid, Clovis, MSD, Pfizer, Roche, Takeda \& Tessa).

Author Contributions

Conceptualization: L.J.Y., K.J.W., L.M.C., P.S.Y., K.B.G.; Data curation: L.J.Y., L.M.C., K.S., K.H.S., Y.J.Y., P.S.Y., K.B.G.; Formal analysis: L.J.Y., K.J.W., K.S., K.H.S., C.C.H.; Funding acquisition: L.J.Y., C.C.H., P.S.Y.; Investigation: L.J.Y., K.J.W., L.M.C., K.S., K.H.S., C.C.H., P.S.Y., K.B.G.; Methodology: L.J.Y., K.J.W., L.M.C., K.S., Y.J.Y., K.B.G.; Project administration: L.J.Y., K.J.W., K.H.S., Y.J.Y., K.B.G.; Resources: L.J.Y., L.M.C., K.S., K.H.S., K.B.G.; Software: L.J.Y., K.J.W., L.M.C., C.C.H., P.S.Y., K.B.G.; Supervision: L.J.Y., K.J.W., C.C.H., K.B.G.; Validation: L.J.Y., K.S., Y.J.Y.; Visualization: L.J.Y., K.J.W., L.M.C., K.H.S., Y.J.Y., P.S.Y.; Writing - original draft: L.J.Y.; Writing - review \& editing: L.J.Y., K.J.W., L.M.C., K.S., K.H.S., C.C.H., Y.J.Y., P.S.Y., K.B.G

\section{INTRODUCTION}

Ovarian cancer is the deadliest gynecological cancer in Korea [1]. The standard therapy is surgical cytoreduction followed by taxane-platinum combination chemotherapy. Several phase 3 clinical trials have demonstrated that survival, postoperative morbidity, and mortality rates after neoadjuvant chemotherapy followed by interval debulking surgery are no worse than those after primary debulking surgery in patients with stages III-IV ovarian cancer [2-4]. Therefore, neoadjuvant chemotherapy followed by interval debulking surgery is an alternative option for patients with unresectable high tumor burden. Most patients with advanced-stage ovarian cancer will achieve complete or partial remission after standard-of-care treatment but eventually will have a relapse. Recent randomized phase 3 studies showed median progression-free survival (PFS) was only 12 months with current chemotherapy in stages III/ IV disease $[2,4]$. Therefore, there is an urgent need to improve the outcomes of patients with this aggressive cancer.

Some recent clinical trials have studied the addition of immune-oncology drugs to current chemotherapy as a front-line treatment. Researchers have especially studied to determine the optimal approach to using immune checkpoint inhibitors. Most strategies considered in front-line settings have been combinations of anti-programmed cell death protein 1 (PD-1) or anti-programmed death-ligand 1 (PD-L1) inhibitors and chemotherapy. IMagyn050 (NCT00262847) is a phase III randomized study designed to compare the efficacy and safety of atezolizumab+paclitaxel+carboplatin+bevacizumab versus placebo+paclitaxel+carboplatin+bevacizumab in 1,300 ovarian cancer patients [5]. JAVELIN OVARIAN 100 (NCT02718417) is a phase III randomized study investigating avelumab in combination with and/or as a maintenance treatment after a regime of carboplatin/paclitaxel chemotherapy in treating 998 ovarian cancer patients. However, when used as part of a planned interim analysis, JAVELIN OVARIAN 100 does not permit having a primary endpoint for PFS.

So far, emerging clinical data shows a limited clinical efficacy of immune checkpoint inhibitors in ovarian cancer; the results have been objective response rates (ORRs) of $10 \%-15 \%$, with some durable responses. Because ovarian cancer is an immunologically cold tumor [6], the use of PD-1 or PD-L1 inhibitors alone likely will have limited benefit. We suggest combination therapy to turn cold tumor into hot tumor. To improve the efficacy of a PD-L1 inhibitor, a combination of a cytotoxic T-lymphocyte-associated protein 4 (CTLA4) inhibitor and chemotherapy has been suggested with promising prospects. NRG GY003 showed that combining nivolumab and ipilmumab was more effective than nivolumab alone (ORR: $31.4 \%$ vs. $12.2 \%$ ) in recurrent ovarian cancer. Korean Gynecologic Oncology Group (KGOG) 3045 is an umbrella study of biomarker-driven targeted therapy in patients with platinum-resistant recurrent ovarian cancer. In this study, one of treatment arms includes durvalumab and tremelimumab plus nonplatinum-based standard of chemotherapy (SOC) (weekly palictaxel, topotecan, or liposomal doxorubicin). In other solid cancers, several ongoing studies such as POSEIDON continue to evaluate the efficacy and safety of the antiPD-L1 and anti-CTLA-4 antibodies plus chemotherapy. In addition, some chemotherapeutic agents induce immunogenic cells and potentially improve the efficacy of immune checkpoint inhibitors [7]. Therefore, we proposed a combination therapy of durvalumab, tremelimumab, and chemotherapy to improve outcomes in advanced-stage ovarian cancer. KGOG 3046 will be uniquely positioned to elucidate whether dual checkpoint inhibition (durvalumab+tremelimumab) with chemotherapy can improve survival without excess toxicity for patients. 
This study protocol was approved by AstraZeneca in June 2018 and the Scientific Review Board of the KGOG in October 2018. Patient enrollment began in June 2019. We obtained approval from the Institutional Review Board before initiating patient accrual at each institution (4-20190083). This trial has been registered with ClinicalTrials.gov NCT03699449.

\section{PROTOCOL OF THE KGOG 3046 STUDY}

\section{Objective}

This single-arm phase II study aims to explore the synergistic effects of durvalumab and tremelimumab plus chemotherapy in advanced-stage ovarian cancer.

The secondary objective is to explore the safety of durvalumab and tremelimumab plus chemotherapy in advanced-stage ovarian cancer.

An exploratory objective is to identify changes in immune biomarkers by comparing tissue and blood samples before and after treatment.

\section{Endpoints}

The primary endpoint of the study is a 12-months PFS rate.

The secondary endpoints include response rates by Response Evaluation Criteria in Solid Tumors version 1.1 after neoadjuvant chemotherapy, immune-related response criteria after neoadjuvant chemotherapy, response rates by PET Response Criteria in Solid Tumors after neoadjuvant chemotherapy, R0 rate at interval debulking surgery, the rate of chemotherapy response scores (CRS) of 3 at interval debulking surgery, overall survival (OS), safety, and duration of response.

PFS is defined as the time from the start of treatment until the first documented sign of disease progression or death from any cause; OS is defined as the time from first treatment until death from any cause. We will estimate PFS and OS using Kaplan-Meier techniques and calculate $95 \%$ confidence intervals.

The following are the details of how (CRS) will be analyzed. An experienced gynecologic pathologist (Prof. Hyun Soo Kim and Sang Yong Song) will score each slide independently according to the 3-tiered CRS system proposed by Bohm et al. Resected specimens from interval debulking surgery will be formalin-fixed and paraffin-embedded according to standard procedures and stained with hematoxylin and eosin stain. The slide with the most viable tumor and/or the least chemotherapy response will be selected from the omentum.

\section{Study design}

Single arm, phase II study.

\section{ELIGIBILITY CRITERIA}

\section{Inclusion criteria}

1) Histologically confirmed adenocarcinoma of ovary, fallopian tube, primary peritoneum (non-mucinous). 
2) Clinical stages IIIC/IV.

3) Eastern Cooperative Oncology Group performance status of $0-1$.

4) Females aged 20 years or older at the time of acquisition of informed consent.

5) Adequate organ function.

6) Body weight $>30 \mathrm{~kg}$.

\section{Exclusion criteria}

1) A serious concomitant systemic disorder that, in the opinion of the investigator, would compromise the patient's ability to complete the study.

2) Patients with active or uncontrolled infections or with serious illnesses or medical conditions.

3) Active pulmonary tuberculosis.

4) Known acute or chronic hepatitis B or C by serological evaluation.

5) Known human immunodeficiency virus infection.

6) History of another primary malignancy that is currently clinically significant or currently requires active intervention.

7) Inability to comply with protocol or study procedures.

\section{RANDOMIZATION}

Not applicable.

\section{TREATMENT}

Patients with histologically confirmed ovarian cancer are offered durvalumab, tremelimumab plus chemotherapy. KGOG 3046 regimen consists of 21-day cycles with durvalumab, tremelimumab plus chemotherapy for neoadjuvant chemotherapy and durvalumab plus chemotherapy for adjuvant chemotherapy (Fig. 1).

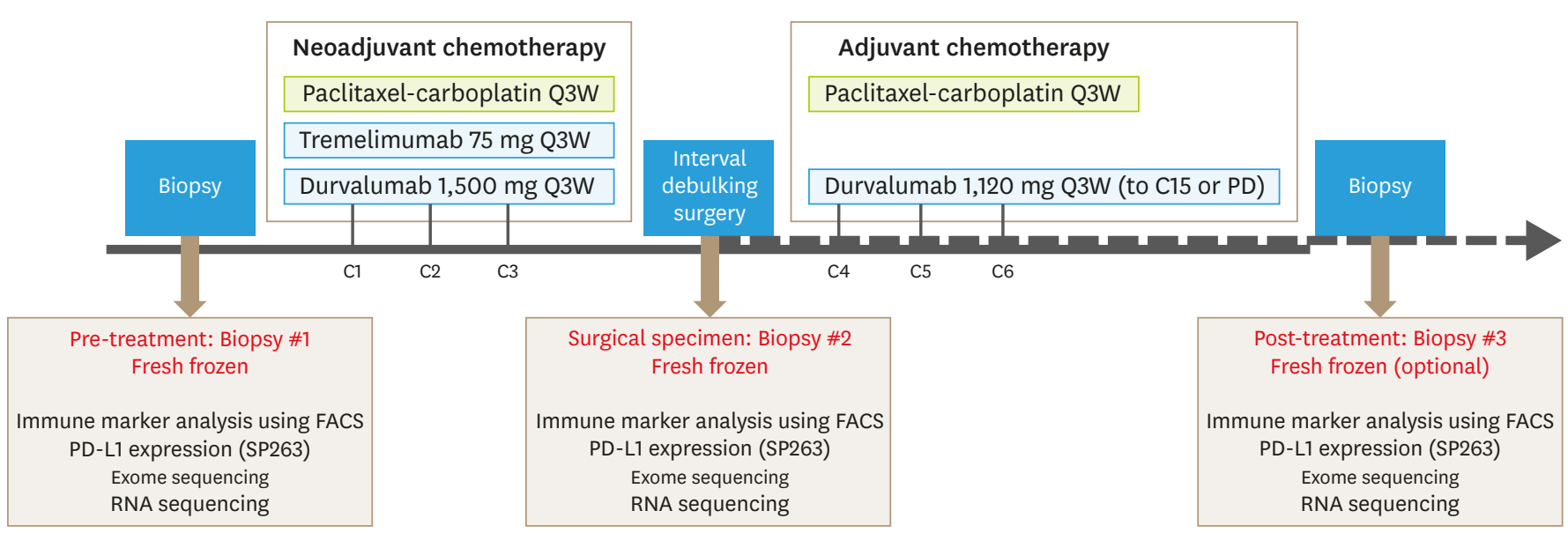

Fig. 1. Study schema.

FACS, fluorescence-activated cell sorting; PD-L1, programmed death-ligand 1; Q3W, every 3 weeks. 


\section{Treatment arm}

Neoadjuvant treatment: standard chemotherapy+durvalumab +tremelimumab.

Durvalumab: 1,500 mg every 3 weeks (Q3W; 3 total doses).

Tremelimumab: 75 mg Q3W (3 total doses).

Chemotherapy regimen: paclitaxel $175 \mathrm{mg} / \mathrm{m}^{2}$, carboplatin area under the curve (AUC) 5-6 Q3W (3 total doses).

Adjuvant treatment: standard chemotherapy+durvalumab.

Durvalumab: 1,120 mg Q3W (12 total doses).

Chemotherapy regimen: paclitaxel $175 \mathrm{mg} / \mathrm{m}^{2}$, carboplatin AUC 5-6 Q3W (3 total doses).

\section{Safety run-in phase}

There will be a safety run-in phase with up to 7 patients. After that, a phase II expansion phase will be conducted with dosage levels considered safe.

Based on historical data, the proportion of patients who experience G3 or more treatmentrelated adverse events (TRAEs) in the neoadjuvant chemotherapy will be approximately $30 \%-50 \%$, even without durvalumab and tremelimumab [8-10].

Enrollment will be suspended until after seven subjects have undergone surgery. Data and Safety Monitoring Board will review the safety of this trial and suggest recommendations, taking into consideration such examples as the following:

If patients who experience grade 3 or more TRAEs (except hematologic toxicity) are $\geq 4$, then the trial will be stopped; If patients who experience grade 3 or more TRAEs (except hematologic toxicity) are $\leq 3$, then the trial will proceed to phase II.

Any recommendations issued as a result of the above situations will be used to decide whether to stop the trial or proceed to phase II.

\section{A single-arm phase II study}

Durvalumab 1,500 mg IV, tremelimumab $75 \mathrm{mg}$ IV, paclitaxel $175 \mathrm{mg} / \mathrm{m}^{2}$, and carboplatin AUC 5 or 6 will be administered every 3 weeks. Three cycles of neoadjuvant chemotherapy will be performed before interval debulking surgery. After surgery, durvalumab 1,120 mg IV, paclitaxel $175 \mathrm{mg} / \mathrm{m}^{2}$, and carboplatin AUC 5 or 6 will be administered every 3 weeks. Three cycles of adjuvant chemotherapy and 12 cycles of durvalumab will be performed after interval debulking surgery.

Treatment response assessments will be implemented every 3 cycles during the treatment period. For patients who achieve disease control after 12 months of treatment, tumor assessments should be performed relative to the date of first infusion as follows: every 12 weeks for the first 2 years, then every 24 weeks thereafter until confirmed PD. All patients will be followed for at least 5 years after treatment is completed. Enhanced abdominal computed tomography and/or magnetic resonance imaging, chest computed tomography, and tumor 
markers will be evaluated at least every 12 weeks until disease progression or death. Efficacy analyses are based on a modified intent-to-treat approach (patients should receive at least one treatment dose).

\title{
SAMPLE SIZE JUSTIFICATION
}

The sample size was calculated based on the 1-sample log-rank test.

Median PFS is 12 months in advanced-stage ovarian cancer from European Organisation for Research and Treatment of Cancer 55971 and Chemotherapy or Upfront Surgery. The rate of patients with disease-free status at 12 months is expected to be $50 \%$ with conventional chemotherapy (SOC). The rate of patients with disease-free status at 12 months is expected to be $70 \%$ with the addition of immunotherapy (combination of durvalumab+tremelimumab+SOC followed by durvalumab+SOC) (hazard ratio $=0.515$ ). With $80 \%$ statistical power and $5 \%$ of 1 -sided type I errors, 21 patients are needed when patients are accrued for 12 months and followed for 30 months after the last patients is enrolled. The number of expected events would be 14. In anticipation of a 10\% drop-out rate, 24 patients will be enrolled in this study.

\section{MONITORING}

The investigator or a designated representative must enter in the electronic case report form all information required by the protocol. The Data and Safety Monitoring Committee will review the results independently from the investigators and the study group statistician. The KGOG Data Center and study coordinator will conduct central monitoring and will issue a monitoring report every 6 months to evaluate study progress and improve data integrity and patient safety. For quality assurance, the KGOG Audit Committee will perform site audits. No interim analysis is planned for this study.

\section{PARTICIPATING INSTITUTIONS}

Yonsei University Severance Hospital, Seoul National University Hospital, Samsung Seoul Medical Center, National Cancer Center.

\section{ACKNOWLEDGMENTS}

This investigator-initiated trial was supported by a faculty research grant of Yonsei University College of Medicine for 6-2018-0169. Research grants are administered by the Ministry of Science, ICT \& Future Planning (2017M3A9E8029714). This trial was also supported by AstraZeneca. We thank AstraZeneca for providing funding and durvalumab/tremelimumab. We appreciate the contribution of investigators and study teams at the participating sites and KGOG.

\author{
Yonsei University Health System, Severance Hospital \\ Eun Ji Nam \\ Sang Wun Kim
}


Kyung Jin Eoh

Yong Jae Lee

Jeongeun Roh

Hye Min Kim

\author{
Sungkyunkwan University Samsung Medical Center \\ Duk-Soo Bae \\ Jeong Won Lee \\ Tae-Joong Kim \\ Yoo Young Lee \\ Eun Hwa Choi \\ SangYong Song \\ Hyun-Soo Kim \\ Seoul National University Hospital \\ Maria Lee \\ Se Ik Kim \\ Eun-Ji Lee \\ Sook-In Kwon \\ National Cancer Center \\ Wonkyo Shin \\ Sang-Soo Seo \\ Sokbom Kang

\section{KGOG} \\ Min Seo Kim \\ Jong Soon Kim \\ Samsung Genome Institute \\ Jegun Joung \\ Jongsuk Chung
}

\title{
REFERENCES
}

1. Lee JY, Kim S, Kim YT, Lim MC, Lee B, Jung KW, et al. Changes in ovarian cancer survival during the 20 years before the era of targeted therapy. BMC Cancer 2018;18:601.

PUBMED | CROSSREF

2. Kehoe S, Hook J, Nankivell M, Jayson GC, Kitchener H, Lopes T, et al. Primary chemotherapy versus primary surgery for newly diagnosed advanced ovarian cancer (CHORUS): an open-label, randomised, controlled, non-inferiority trial. Lancet 2015;386:249-57. PUBMED | CROSSREF

3. Onda T, Satoh T, Saito T, Kasamatsu T, Nakanishi T, Nakamura K, et al. Comparison of treatment invasiveness between upfront debulking surgery versus interval debulking surgery following neoadjuvant chemotherapy for stage III/IV ovarian, tubal, and peritoneal cancers in a phase III randomised trial: Japan Clinical Oncology Group Study JCOG0602. Eur J Cancer 2016;64:22-31. PUBMED | CROSSREF

4. Vergote I, Tropé CG, Amant F, Kristensen GB, Ehlen T, Johnson N, et al. Neoadjuvant chemotherapy or primary surgery in stage IIIC or IV ovarian cancer. N Engl J Med 2010;363:943-53. PUBMED | CROSSREF 
5. Moore KN, Pignata S. Trials in progress: IMagyn050/GOG 3015/ENGOT-OV39. A Phase III, multicenter, randomized study of atezolizumab versus placebo administered in combination with paclitaxel, carboplatin, and bevacizumab to patients with newly-diagnosed stage III or stage IV ovarian, fallopian tube, or primary peritoneal cancer. Int J Gynecol Cancer. Forthcoming 2019. PUBMED

6. Kim HS, Kim JY, Lee YJ, Kim SH, Lee JY, Nam EJ, et al. Expression of programmed cell death ligand 1 and immune checkpoint markers in residual tumors after neoadjuvant chemotherapy for advanced high-grade serous ovarian cancer. Gynecol Oncol 2018;151:414-21. PUBMED | CROSSREF

7. Galluzzi L, Buqué A, Kepp O, Zitvogel L, Kroemer G. Immunological effects of conventional chemotherapy and targeted anticancer agents. Cancer Cell 2015;28:690-714. PUBMED | CROSSREF

8. Becker DA, Thomas ED, Gilbert AL, Boone JD, Straughn JM Jr, Huh WK, et al. Improved outcomes with dose-dense paclitaxel-based neoadjuvant chemotherapy in advanced epithelial ovarian carcinoma. Gynecol Oncol 2016;142:25-9. PUBMED | CROSSREF

9. Garcia YG, Juan AD, Mendiola C, Barretina-Ginesta MP, Prat A, Santaballa A, et al. Phase II randomized trial of neoadjuvant (NA) chemotherapy (CT) with or without bevacizumab (Bev) in advanced epithelial ovarian cancer (EOC) (GEICO 1205/NOVA TRIAL). 2017 ASCO Annual Meeting; 2017 Jun 2; Chicago, IL: American Society of Clinical Oncology; 2017. Abstract 5508.

10. Yoshihama T, Nomura H, Iwasa N, Kataoka F, Hashimoto S, Nanki Y, et al. Efficacy and safety of dosedense paclitaxel plus carboplatin as neoadjuvant chemotherapy for advanced ovarian, fallopian tube or peritoneal cancer. Jpn J Clin Oncol 2017;47:1019-23.

PUBMED | CROSSREF 